\title{
STABLE HOMOTOPY THEORY OVER A FIXED BASE SPACE
}

\author{
BY C. A. ROBINSON
}

Communicated by Glen E. Bredon, August 30, 1973

A spectral sequence which may be regarded as an 'Adams spectral sequence over a fixed space $B$ ' has been constructed by J. F. McClendon [6] and J.-P. Meyer [8]. This note describes a generalization in which there is no need for any orientability assumptions. The construction is carried out in a suitable stable category, which may be of independent interest. An application to the enumeration of immersions is given. Details will appear elsewhere.

1. A stable category. Let $E x-B$ denote the category of ex-spaces (in the terminology of [4]) of the path-connected complex $B$. It is known that the corresponding homotopy category $(E x-B)_{h}$ exhibits certain stability properties [4, Theorem 6.4]. One can construct a category $\mathscr{S} \mid B$, in which the corresponding stable homotopy theory can be investigated, by formalizing the notion of a 'bundle' over $B$ with fibre a $C W$ spectrum (in the sense of [3], [11]). The details are as follows. If $F_{1}, F_{2}, F$ are objects of the category $\mathscr{S}$ of $C W$ spectra, there is a simplicial set of morphisms $\operatorname{Mor}_{\mathscr{S}}\left(F_{1}, F_{2}\right)$; and $\operatorname{Mor}_{\mathscr{S}}(F, F)$ is a simplicial monoid whose invertible elements form a simplicial group Aut $_{\mathscr{S}} F$. We take $B$ to be a simplicial set rather than a space.

Definition. An object of $\mathscr{S} \mid B$ is a pair $(F, \xi)$ where $F \in$ ob $\mathscr{S}$ and $\xi$ is a principal simplicial Aut $_{\mathscr{S}} F$-bundle over $B$.

A morphism from $\left(F_{1}, \xi_{1}\right)$ to $\left(F_{2}, \xi_{2}\right)$ is a section of the simplicial bundle with fibre $\operatorname{Mor}_{\mathscr{S}}\left(F_{1}, F_{2}\right)$ associated to the principal (Aut ${ }_{\mathscr{S}} F_{1} \times \operatorname{Aut}_{\mathscr{S}} F_{2}$ )bundle $\xi_{1} \times \xi_{2}$ over $B$.

This category inherits much of the usual machinery of stable homotopy theory from Boardman's category $\mathscr{S}$; for example, it has an invertible translation-suspension functor $S_{B}$, arbitrary wedges, and smash-product functors. The corresponding homotopy category $(\mathscr{S} \mid B)_{h}$ is additive, and triangulated with respect to $S_{B}$, and the axioms of Puppe [9] hold. There is a stabilization functor $(E x-B)_{h} \rightarrow(\mathscr{S} \mid B)_{h}$ which is bijective on morphismsets $[X, Y]_{B}$ of $(E x-B)_{h}$ whenever $X$ is a relative $C W$ ex-space of $B, Y$ is an ex-space fibred over $B$ (with fibre $F$, say) and $\operatorname{dim}(X-B) \leqq 2$ conn $F$.

2. A cohomology theory on $\mathscr{S} \mid B$. Let $p$ be a prime, and let $\rho: \pi_{1} B \rightarrow$ $G L(V)$ be a semisimple representation of $\pi_{1} B$ on a finite-dimensional vector

AMS (MOS) subject classifications (1970). Primary 55H15; Secondary 55B25, 55G35, 57D40. 
space $V$ over $Z / p Z$. Then $\rho$ gives rise to a system of twisted coefficients on $B$, and hence to a cohomology theory on $E x-B$. We define a corresponding cohomology functor on $\mathscr{S} \mid B$ by constructing a representing object $K(\rho) \in \operatorname{ob}(\mathscr{S} \mid B)$. This object is a 'bundle' with fibre the EilenbergMac Lane spectrum $K(V) \in \mathrm{ob} \mathscr{S}$, and is a stable version of the EilenbergMac Lane bundles in [10].

As the analogue 'over $B$ ' of the Steenrod algebra $A_{p}^{*}$, we obtain a graded abelian category $A_{B, p}^{*}$ as follows. The objects are the finite-dimensional, semisimple representations of $\pi_{1} B$ over the field $Z / p Z$ : the morphisms from a representation $\rho$ to a representation $\sigma$ form the graded abelian group $\{K(\rho), K(\sigma)\}_{B}^{*}$, where $\{,\}_{B}^{*}$ denotes graded homotopy classes in $\mathscr{S} \mid B$. Composition is evident. We regard $A_{B, p}^{*}$ as a 'ring with several objects', and introduce the corresponding abelian category of graded left modules $A_{B, p}^{*}$-mod: this is just the category of additive functors from $A_{B, p}^{*}$ to the category of graded abelian groups.

The functors on $\mathscr{S} \mid B$ represented by the various $K(\rho)$ now unite to give a cohomology functor

$$
\boldsymbol{H}^{*}(; p):(\mathscr{S} \mid B)_{h} \rightarrow A_{B, p^{-}}^{*} \text { mod } .
$$

When $B$ is simply-connected, $A_{B, p}^{*}-\bmod$ is equivalent to the category of graded left modules over the Massey-Peterson semitensor algebra $H^{*}(B ; Z / p Z) \circ A_{p}^{*}[7]$.

3. A spectral sequence. We wish to use the foregoing cohomology theory to construct an Adams spectral sequence for $\{X, Y\}_{B}^{*}$, where $X$ and $Y$ are bundles of spectra over $B$. As in the classical Adams spectral sequence, it is necessary to impose some finiteness assumption on $X$, and finite type assumption on $Y$, to ensure existence and convergence of the spectral sequence. We shall assume the following:

(i) $\pi_{1} B$ has only finitely many distinct irreducible finite-dimensional representations over $Z / p Z$.

(ii) For each such irreducible $\rho$, the groups $H^{*}(B ; \rho)$ and $H^{*}(Y ; \rho)$ are finitely-generated in each dimension.

(iii) The fibre-spectrum $F$ of $Y$ is highly-connected, and $\pi_{*} F$ is finitelygenerated in each dimension.

(iv) $X$ is the image in $\mathscr{S} / B$ of a finite (relative) $C W$ ex-space of $B$.

Under these conditions, there is a diagram in $\mathscr{S} \mid B$ with exact triangles (dotted morphisms have degree -1 )

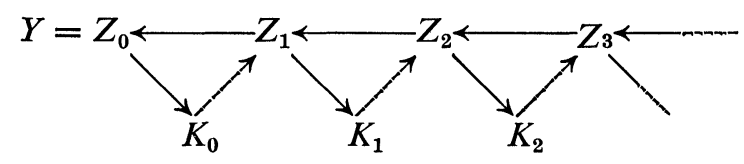


such that the induced cohomology diagram

$$
\boldsymbol{H}^{*}(Y ; p) \leftarrow \boldsymbol{H}^{*}\left(K_{0} ; p\right) \leftarrow \boldsymbol{H}^{*}\left(K_{1} ; p\right) \leftarrow \cdots
$$

is a minimal free resolution of $\boldsymbol{H}^{*}(Y ; p)$ in $A_{B, p}^{*}$-mod. We now obtain a Cartan-Eilenberg system by applying the functor $\{X,\}_{B}^{*}$, and hence a spectral sequence.

THEOREM. Under the conditions (i)-(iv) above, this spectral sequence has

$$
E_{2}^{s, t} \approx \operatorname{Ext}_{A_{B, p}^{s}, t \text { mod }}^{s, t}\left(H^{*}(Y ; p), H^{*}(X ; p)\right)
$$

and converges to the quotient of $\{X, Y\}_{B}^{*}$ by the subgroup of torsion elements of order prime to $p$.

The proof uses the existence of nonsimple modified Postnikov towers [10], and otherwise follows the convergence proof of [1].

Smash- and composition-product pairings can be introduced into the spectral sequence. The former can be used to give it the structure of a module over the usual Adams spectral sequence for the stable stems.

4. The enumeration of immersions. By the immersion theory of $M$. Hirsch, the regular homotopy classes of smooth immersions of a smooth manifold $M^{m}$ in $R^{m+n}$ are enumerated for $n>1$ by liftings in the diagram

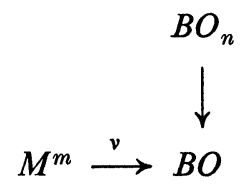

where $v$ represents the stable normal bundle. According to a theorem of J. C. Becker [2], in the case $m \leqq 2 n-2$ these liftings are enumerated by difference classes in a certain stable track group in $E x-B O$, which becomes a morphism group in $(\mathscr{S} \mid B O)_{h}$ and hence can be calculated from the spectral sequence. Calculation with specific resolutions for the spectrum bundle obtained from $B O_{n} \rightarrow B O$ yields the following results for real projective spaces.

THEOREM. Suppose $P^{m}$ immerses in $R^{2 n-k}$, where $0 \leqq k \leqq 5$ and $(m-k) \geqq$ 7. Then the difference group for such immersions is as given in Table 1 . In particular, the number of regular homotopy classes is given by the order of the appropriate group.

This theorem extends special cases of results of I. M. James and P. E. Thomas [5], and appears to agree with recent calculations of H. A. Salomonsen, who uses a more geometric approach. The ambiguities in the table are due to undetermined higher differentials. 


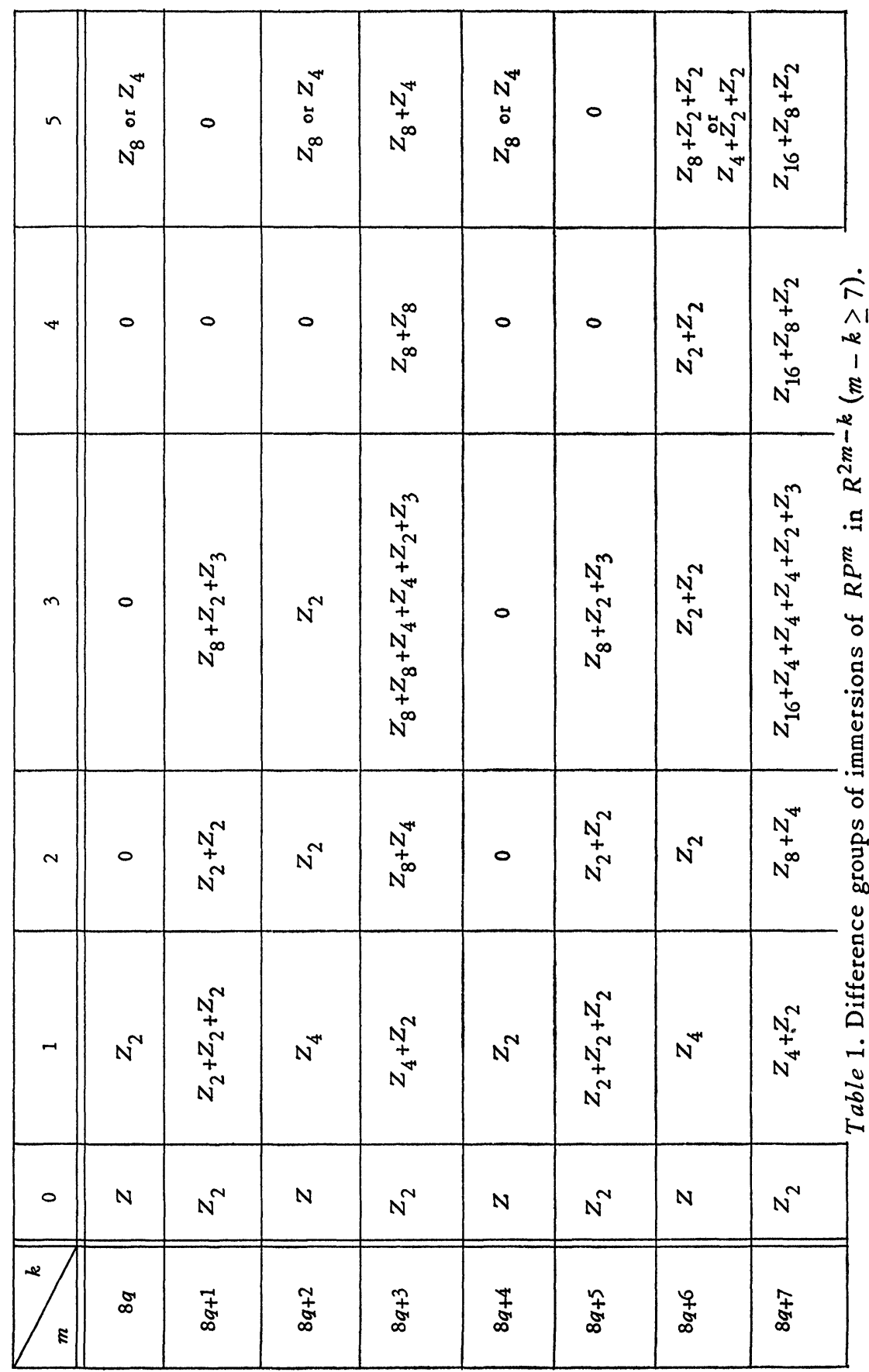




\section{REFERENCES}

1. J. F. Adams, On the structure and applications of the Steenrod algebra, Comment. Math. Helv. 32 (1958), 180-214. MR 20 \#2711.

2. J. C. Becker, Cohomology and the classification of liftings, Trans. Amer. Math. Soc. 133 (1968), 447-475. MR 38 \#5217.

3. J. M. Boardman, Stable homotopy theory, University of Warwick (duplicated typescript), 1965.

4. I. M. James, Bundles with special structure. I, Ann. of Math. (2) 89 (1969), 359-390. MR 39 \#4868.

5. I. M. James and P. E. Thomas, On the enumeration of cross-sections, Topology 5 (1966), 95-114. MR 33 \#4939.

6. J. F. McClendon, A spectral sequence for classifying liftings in fiber spaces, Bull. Amer. Math. Soc. 74 (1968), 982-984. MR 38 \#2781.

7. W. S. Massey and F. P. Peterson, The cohomology structure of certain fibre spaces. I, Topology 4 (1965), 47-65. MR 32 \#6459.

8. J.-P. Meyer, Relative stable homotopy, Proc. Conf. on Algebraic Topology, University of Illinois at Chicago Circle, 1968, pp. 206-212.

9. D. Puppe, On the formal structure of stable homotopy theory, Proc. Colloq. on Algebraic Topology, University of Aarhus, 1962, pp. 65-71.

10. C. A. Robinson, Moore-Postnikov systems for non-simple fibrations, Illinois J. Math. 16 (1972), 234-242. MR 45 \#7714.

11. R. M. Vogt, Boardman's stable homotopy category, Lecture Note Series, no. 21, Matematisk Institut, Aarhus Universitet, Aarhus, 1970. MR 43 \#1187.

Mathematics Institute, University of Warwick, Coventry, England. 